\title{
The Trend of Sickness Absenteeism among Health Care Workers
}

\section{Nazanin I* and Maryam M}

Center for Research on Occupational Disease, Tehran University of Medical Sciences, Tehran, Iran

*Corresponding author: Nazanin Izadi, Associate professor of occupational medicine, MD, Center for Research on Occupational Disease, Tehran University of Medical Sciences, Tehran, Iran, Tel: 0098(021) 66405588; Email: Nazanin.izadi@gmail.com

\section{Research Article}

Volume 4 Issue 1

Received Date: January 20, 2020

Published Date: March 03, 2020

DOI: $10.23880 /$ phoa- 16000150

\section{Abstract}

Background: Sickness absence is an important issue in the hospitals. The aim of this study was to investigate sickness absenteeism trend during 3 years after implementing occupational health office.

Material and Method: This study was conducted using sickness absenteeism data of hospital staff from a teaching hospital in Tehran from 2014 to 2016. Demographic and occupational characteristics were measured by a questionnaire survey.

Results: In our study, mean days of absence was 11.64 and 508 episodes occurred during the 3 years. There was a decreasing trend of all sickness absence parameters during the study period. The most common causes of sickness absence were respiratory problems and musculoskeletal disorders.

Conclusion: The study abounds in the implications of active workplace intervention such as implementing a monitoring system based on scientific guidelines such as an occupational health office, providing principles of infection control program and ergonomic interventions are effective in reducing sickness absence.

Keywords: Sickness Absence; Health Care Worker; Occupational Health

\section{Introduction}

Sickness absence is defined as an absence from work due to sickness or injury accepted by the employer or the social security system [1]. Absenteeism is a complex phenomenon which could have different predictors in each organization. Excessive sickness absenteeism might be shown that there is something wrong in the person-work-society relationship $[1,2]$. Sickness absence is of great concern in hospitals because health care workers face a wide range of hazards on the job, including exposure to blood and body fluids, chemical hazards, stress and violence and ergonomic hazards, therefore a manpower shortage may easily disrupt service delivery, increases work load and negatively affects patient safety [3].
Although sickness absence is seen as a major health problem in health care settings, little is known about the sickness absence frequency and contributing factors in developing countries.

In Iranian hospitals, health care workers report sick to their supervisor, when they couldn't attend work because of illness, then the employee should provide a medical certificate to one of the approved doctors of the hospital, and usually these certificates signed and approved with no concern and attitude about returning to work limitations. It seems that the effectiveness of this approach is not satisfactory to the employer and organization. After implementing occupational health clinic in the hospital, we decided to assess the role of this clinic in reporting and controlling sickness absenteeism of staffs. 
According to our knowledge, there is no study dealing about sickness absenteeism among hospital staffs in Iran. This study was carried out to investigate sickness absenteeism in health care workers of a teaching hospital during 3 years so we could look a trend of sickness absence at this hospital by comparing the data after implementing occupational health clinic with the previous data, also describes the sickness absenteeism frequency, main involved disease and associated factors.

\section{Materials and Methods}

This before- after study was conducted using sickness absenteeism data of health care workers Baharloo hospital of Tehran University of Medical Sciences. Bahrloo hospital was the first hospital, which has the most developed occupational medicine clinic in Iran and was the first hospital having a comprehensive program of medical surveillance of health care workers to minimize adverse health effects due to their potential exposure to different types of occupational hazards [4].

In Iran a medical certificate is mandatory for any kind of sick leave, and the compensation is equal to the normal salary during this period. The sick leave was approved by the attending doctor of the hospital before October 2014, after that date the manager decided to change the sick leave policy under the supervision of occupational health clinic, that every report should be signed and approved by the occupational medicine specialist according to the return to work guidelines [5].

The study reviewed all sickness absence leave over a period of three consecutive years from 23 September 2014 to 23 September 2016. Data records include demographic characteristics (age, sex, marital status, number of children, educational level), occupational factors (employment type, job type, ward, shift work, duration of employment), diagnosis, spells and duration of sick leave. For the purpose of the study, sick leave patterns were measured in terms of sickness absence rate (SAR); total number of certified sickness absence days per person year and absence frequency rate (AFR); total number of sickness absence spells per total employees. AFR was categorized as no absence and $\geq 1$ sick leave episodes. Total days lost by type of sickness: total days lost due to cause A per total days lost from all causes and. Average days lost per worker: total days lost due to sickness per average number of workers. Working days in the study period was 290 days. Maternity or other kinds of parental leave were excluded from our study.

All physician-certified data were gathered through computerized records in the occupational health clinic then were entered into SPSS software version 18 and got analyzed using descriptive and analytic statistics. In order to evaluate the association of demographic and occupational factors that can affect sickness absence among hospital staffs Ethical approval was obtained from the Tehran University of Medical Sciences, data were entered anonymously.

\section{Results}

According to the department of human resources, total number of hospital employees during the three year study period ranged from 423 to 609 subjects. The mean (SD) age and working years of the study population were 34.74(8.22) and 7.52(5.8) years, respectively. Two-third of employees was female and half of them had a bachelor degree or more. Data on the distribution of the HCWs according to demographic and occupational characteristics has been presented in Table 1. Using non parametric analysis (Mann Whitney test) there was no statistical significance between demographic and occupational characteristics and median days of sick leave, however, we have seen that having children, shift-workers and contact employers were associated with more absence spells, but we found no correlation in logistic regression model (Table 1).

\begin{tabular}{|c|c|c|c|c|c|c|}
\hline \multirow{3}{*}{\multicolumn{2}{|c|}{ Variables }} & \multirow{3}{*}{ n (\%) } & \multirow{3}{*}{$\begin{array}{c}\text { Sickness } \\
\text { absence days } \\
\text { Median (IQR) }\end{array}$} & \multicolumn{2}{|c|}{ Sickness absence episode } & \multirow{3}{*}{ OR (CI95\%) } \\
\hline & & & & $\leq 1$ & $>1$ & \\
\hline & & & & $n(\%)$ & $n(\%)$ & \\
\hline \multirow{2}{*}{ Age } & $<35$ & $199(58.2)$ & $4(2-12)$ & $94(47.2)$ & $105(52.8)$ & 1.2 \\
\hline & $>=35$ & $143(41.8)$ & $5(2-15)$ & $61(42.7)$ & $82(57.3)$ & $(0.78-1.85)$ \\
\hline \multirow{2}{*}{ Sex } & Male & $75(21.9)$ & $4(2-14)$ & $40(53.3)$ & $35(46.7)$ & 1.51 \\
\hline & Female & $267(78.1)$ & $4(2-14)$ & $115(43.1)$ & $152(56.9)$ & $(0.9-2.52)$ \\
\hline \multirow{2}{*}{ Marital status } & Single & $113(33)$ & $4(2-11)$ & $57(50.4)$ & $56(49.6)$ & 1.3 \\
\hline & Married & $229(67)$ & $5(2-14)$ & $98(42.8)$ & $131(57.2)$ & $(0.86-2.13)$ \\
\hline
\end{tabular}


Public Health Open Access

\begin{tabular}{|c|c|c|c|c|c|c|}
\hline \multirow{2}{*}{ Children } & No children & $165(48.5)$ & $4(2-11)$ & $84(50.9)$ & $81(49.1)$ & $1.55^{*}$ \\
\cline { 2 - 7 } Educational level & $\geq 1$ Children & $175(51.5)$ & $5(2-15)$ & $70(40)$ & $105(60)$ & $(1.71-2.39)$ \\
\hline \multirow{2}{*}{ Employment type } & <bachelor & $108(31.6)$ & $4(2-14)$ & $55(50.9)$ & $53(49.1)$ & 1.3 \\
\cline { 2 - 7 } & $>=$ bachelor & $234(68.4)$ & $4(2-14)$ & $100(42.9)$ & $134(57.3)$ & $(0.88-2.19)$ \\
\cline { 2 - 7 } & Contract & $178(52.2)$ & $4(2-12.5)$ & $91(51.1)$ & $87(48.9)$ & $1.61^{*}$ \\
\hline \multirow{3}{*}{ Job } & Formal & $163(47.8)$ & $4(2-17)$ & $64(39.3)$ & $99(60.7)$ & $(1.05-2.48)$ \\
\hline \multirow{3}{*}{ Ward } & $\begin{array}{c}\text { Nursing } \\
\text { group }\end{array}$ & $209(61.1)$ & $4(2-13.5)$ & $98(46.9)$ & $111(53.1)$ & 1.17 \\
\cline { 2 - 7 } & Others & $133(38.3)$ & $5(2-14)$ & $57(42.9)$ & $76(57.1)$ & $(0.76-1.82)$ \\
\cline { 2 - 7 } & $\begin{array}{c}\text { ICU,CCU, } \\
\text { emergency }\end{array}$ & $132(38.6)$ & $4(2-11)$ & $66(50)$ & $66(50)$ & 1.36 \\
\hline \multirow{2}{*}{\begin{tabular}{c} 
Shift work \\
\cline { 2 - 7 }
\end{tabular}} & Rotatory & $165(48.4)$ & $4(2-13)$ & $85(51.5)$ & $80(48.5)$ & $1.64^{*}$ \\
\cline { 2 - 7 } & Fix & $176(51.6)$ & $5(2-14)$ & $69(39.2)$ & $107(60.8)$ & $(1.07-2.53)$ \\
\hline Duration of & $<5$ years & $218(63.7)$ & $4(2-11)$ & $106(48.6)$ & $112(51.4)$ & 1.44 \\
\cline { 2 - 7 } & $>=5$ years & $124(36.3)$ & $6(2.25-18)$ & $49(39.5)$ & $75(60.5)$ & $(0.92-2.26)$ \\
\hline
\end{tabular}

Table 1: Demographic and Occupational Characteristic of Our Study Population According to Median Days and Episodes of Sick Leave.

${ }^{*} \mathrm{P}$ - value $<0.05$ was considered significant.

In our study, mean days of absence was 11.64 and 508 episodes occurred during the 3 years. Short-term sickness absence ( $\leq 3$ days) was $326(64.2 \%)$ and Long-term sickness absence ( $>4$ days) was 182(35.8\%). Comparison of sickness absence days, according to first medical certificates and certificates approved by occupational health clinic during three years is shown in Table 2. Figure 1 presented decreasing trend of all sickness absence parameters during the study period. The most common causes of sickness absence were respiratory problems (64.7\%), musculoskeletal disorders $(22.2 \%)$, injury $(16.9 \%)$ and infectious disease $(13.5 \%)$ according to distribution of spells attributed to each diagnostic group (Figure 2).

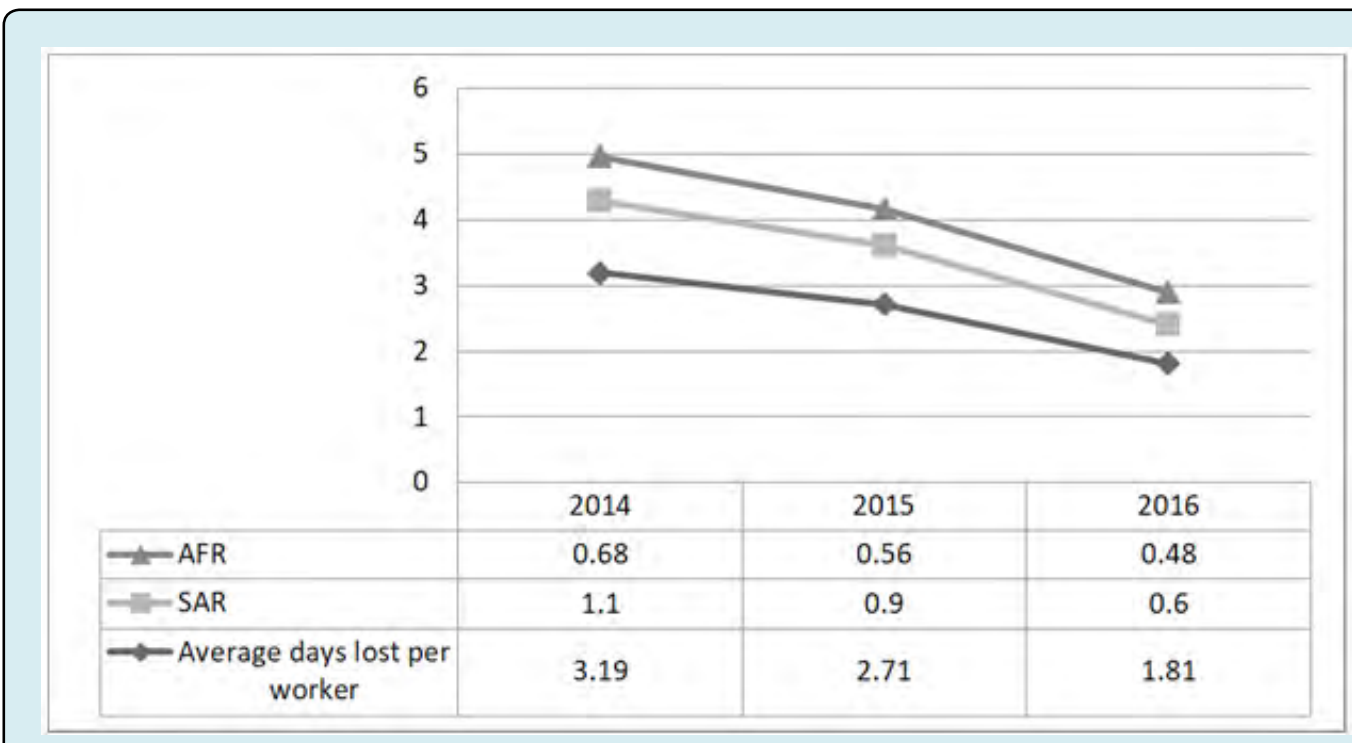

Figure1: Sickness absenteeism parameters in our study population during three years. 


\begin{tabular}{|c|c|c|c|c|c|c|}
\hline Year & & Sum & Mean \pm SD & $\mathbf{t}$ & p-value & \% change \\
\hline \multirow{2}{*}{2014} & First medical certificate for $\mathrm{SA}^{*}$ & 1351 & $8.39 \pm 17.35$ & \multirow{2}{*}{ - } & \multirow{2}{*}{ - } & \multirow{2}{*}{-} \\
\hline & Approved by $\mathrm{OHC}^{\#}$ & 1351 & $8.39 \pm 17.35$ & & & \\
\hline \multirow{2}{*}{2015} & First medical certificate for SA & 1568 & $8.66 \pm 15.8$ & \multirow{2}{*}{2.14} & \multirow{2}{*}{0.033} & \multirow{2}{*}{$2.70 \%$} \\
\hline & Approved by OHC & 1526 & $8.43 \pm 15.56$ & & & \\
\hline \multirow{2}{*}{2016} & First medical certificate for SA & 1148 & $7.30 \pm 11.55$ & \multirow{2}{*}{2.81} & \multirow{2}{*}{0.006} & \multirow{2}{*}{$3.80 \%$} \\
\hline & Approved by $\mathrm{OHC}$ & 1104 & $6.65 \pm 10.23$ & & & \\
\hline
\end{tabular}

Table2: comparison sickness absence days according to different certificates during three years (pair t- test).

"Sickness absence.

\#Occupational Health Clinic.

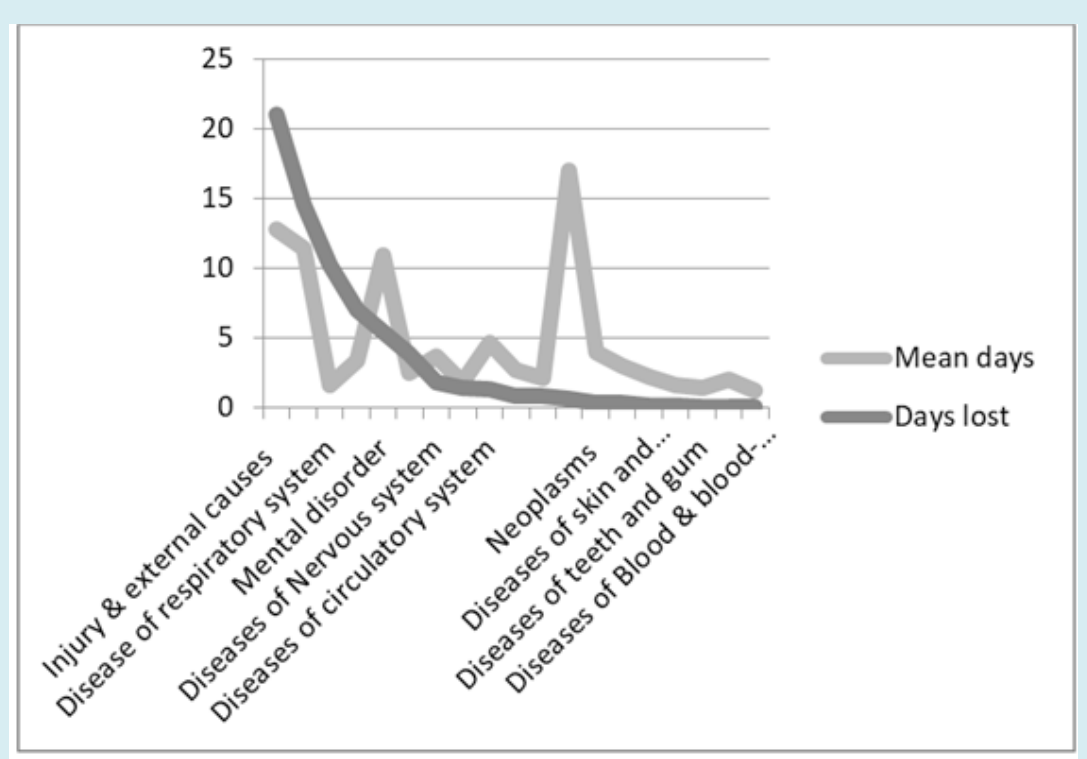

Figure 2: mean days lost and total days lost by cause of sickness absence.

\section{Discussion}

Our study found no association between sickness absence and demographic and occupational factors. This is similar to Khawaja [6] study and against Saravi BM survey [7]. Sickness absence was not associated with either gender or nationality in Rajab Ali Khawaja study [6]. At Saravi BM survey, higher sick leave in female, married, more than bachelor degree and contract employees [7]. We expect that sickness absence to be related to physical and mental health rather than the demographic and occupational characteristics. The global absenteeism rate is more associated with demographic and occupational factors, and usually a U-shaped association was identified between some of these variables, For instance, Absenteeism is more common in young employees with low duration of employment because they have less dependency and responsibility to their jobs inverse old employees with high duration of employment [8].
Sickness absence parameters contain SAR, AFR and average days lost per worker had a decreasing trend during 3 years. The National Health Service reported average annual SAR by $4.2 \%$ for all NHS hospital staff, $4.5 \%$ of nursing group, and $5.5 \%$ of ambulance worker [9]. At Krane, et al. study in the health care sector, was a significant increasing trend in the sickness absence rates in Denmark, but not in Norway during 5 years monitoring [10]. Overall absenteeism rate due to illness was $2.8 \%$ among employees of the Sao Paulo Health Department in Sala study [1]. In our study, sickness absence parameters were less than other studies even before implementing occupational health clinic. This finding may be the following reasons: Strict approval of medical certification, take easily paid leave that preferred to use paid leave instead of sick leave and high workload and responsibility in the workplace. 
The most common causes of sickness absence in our study were respiratory disease and musculoskeletal disorders. Among the respiratory diseases, 214(65.04\%) persons were infected with flu and among musculoskeletal disorder, 69(61.06\%) persons had low back pain. The most common causes of sickness absence at Khawaja study were acute upper respiratory infection, diseases of the musculoskeletal system and disease of the digestive system [6]. In Saravi study, common causes for sickness absenteeism were respiratory disease (35.5\%), skeletal disease (17.7\%), sign and symptom (14.7\%) and infectious disease (11.2\%). Among the respiratory diseases, 115 persons had got cold and 97 were infected with flu [7]. The main known causes of sickness absence on 12900 NHS staff were respiratory, digestive and musculoskeletal disorders respectively [6]. Across all UK workers, common colds, were the most common reason for sickness absence (25\%), the second reasons were musculoskeletal problems (22\%) [9]. In our study, respiratory disease related absence such as flu was a major contributor to all sickness absence, we can conclude that factors such as flu vaccine injection, providing principles of personal protection and infection control can reduce sickness absence due to respiratory and infectious diseases, in regard to frequency of flu vaccine injection less than $50 \%$ in this and other hospitals [4].

A cross-sectional study that was conducted in 2009 at a university hospital, a total of 377 (12.1\%) employees had 416 spells of sickness absence with 639 sick-off days. Mean days were 1.7 and mean episode was 1.1 [6]. A descriptive and cross-sectional study, which was done in 2010 at Mazandaran University of Medical Sciences. Based on the results, 1200 employees were left the organization due to the sickness issue, which 957 (79.7\%) of them were studied. Also, total average sick leave days and total sick leave days were $2 \pm 1$ and 2571 [7]. In Pines survey, mean days were 10.6-11.5 [11]. Mean number of sick leave episodes increased significantly during the study period, from 1.8 to 2.3 in Norway and in Denmark, from 2 to 2.2 in Krane study [10]. In our study, mean days of sickness absence were more than other studies because most studies have been done on short-term sickness absenteeism.

Based on these findings, it seems that active workplace interventions such as implementing a monitoring system based on scientific guidelines such as an occupational health office is effective in reducing sickness absence, also providing principles of infection control program and ergonomic interventions can reduce SAR due to viral respiratory infections and musculoskeletal problems, respectively. The strength of our study is that we used to register sickness absence data, thus vanished measurement error and recall bias. The other strength of this study was all medical certificates approved by occupational health clinic, according to the return to work guidelines and this study was practical for managers and organizations.

The limitation of this study is that done in one hospital, therefore, its' extension to all HCWs is limited. The other limitation is study design, if study was designed cohort or interventions that possible confounding factors were controlled. In regard to ethical consideration, we accept medical certificates for few physicians about psychiatry and maternal problems.

\section{Conflict of Interest}

This study was conducted with the support of Tehran University of Medical Sciences and there is no conflict of interest with any stakeholders. This study did not have any source of financial support.

\section{References}

1. Sala A, Carro AR, Correa AN, Seixas PH (2009) Sick leave among employees of the Sao Paulo State Health Department, Brazil, 2004. Cad Saude Publica 25(10): 2168-2178.

2. Lund T, Labriola M (2009) Sickness absence in Denmarkresearch, results, and reflections. Scan J Work Environ Health Suppl (7): 5-14.

3. Szucs TD, Kristin LN (2006) Economic and social impact of epidemic and pandemic influenza. Vaccine 24(44-46): 6776-6778.

4. Izadi N, Sadeghniiat HK, Malek M (2015) The Results of Medical Surveillance of Health Care Workers by the First Hospital Occupational Health Clinic. J Mil Med 17(2): 7379.

5. Talmage JB, Melhorn M (2005) A physician's guide to return to work. American medical association. AMA press.

6. Khawaja RA, Sikander R, Khawaja AA, Jareno RJ, Halepota AT (2012) Medically certified sickness absence among health care workers. J Pak Med Assoc 62(9): 900-904.

7. Saravi MB, Kabirzadeh A, Rezazadeh E, Khariki MF, Asgari Z, et al. (2013) Prevalence and Causes of Medical Absenteeism Among Staff (Case Study at Mazandaran University of Medical Sciences 2009-2010). Mater Sociomed 25(4): 233-237.

8. Vahtera J, Kivimaki M, Pentti J (2001) The role of extended weekends in sickness absenteeism. Occup Environ Med 58(12): 818-822. 
9. Moberly $\mathrm{T}$ (2018) Sickness absence rates across the NHS. BMJ 361: k2210.

10. Krane L, Johnsen R, Fleten N, Nielsen CV, Stapelfeldt CM, et al. (2014) Sickness absence patterns and trends in the health care sector: 5-year monitoring of female municipal employees in the health and care sectors in
Norway and Denmark. Hum Resour Health 12: 37.

11. Pines A, Skulkeo K, Pollak E, Peritz E, Steif J (1985) Rates of sickness absenteeism among employees of a modern hospital: the role of demographic and occupational factors. Br J Ind Med 42(5): 326-335. 